\title{
AI 驱动的光通信专题简介
}

\section{纪越峰 ${ }^{*}$ ，吕超 ${ }^{2}$, Darko ZIBAR ${ }^{3}$ ，邢焕来 ${ }^{4}$}

1. 北京邮电大学, 北京 100876 , 中国

2. 香港理工大学, 香港 999077 , 中国

3. Technical University of Denmark, Lyngby 2800, Denmark

4. 西南交通大学, 成都 611756 , 中国

* 通信作者. E-mail: jyf@bupt.edu.cn

随着人工智能 (artificial intelligence, $\mathrm{AI}$ ) 等新技术的引入, 给光通信带来了新的活力, 赋予了新的 含义, 形成了新的体系, 构建了新的业态, 受到来自学术界和工业界持续而广泛的关注与极大的研究热 情. 特别是将 AI 与已有光通信系统与网络中的光信号处理等技术相结合, 可为宽带光接入、高速光传 输、灵活光交换、智能光联网等性能提升和网络优化提供高效的解决方案. 然而, AI 在光通信中的研 究与应用尚处起步阶段, 在科研、开发与应用方面仍面临诸多亟待解决的挑战问题.

为共同探讨 $\mathrm{AI}$ 驱动的光通信技术的研究方向, 展现当前研究人员在相关领域的最新研究进展, SCIENCE CHINA Information Sciences 在 2020 年 63 卷第 6 期组织出版了 “AI 驱动的光通信专 题” (Special Focus on Artificial Intelligence for Optical Communications). 经过严格的同行评议, 专题共 收录 5 篇文章, 主题涵盖新型的光网络架构、光信号处理、光参量优化、光传输算法等方面的最新研 究内容与研究成果.

"Artificial intelligence-driven autonomous optical networks: 3S architecture and key technologies" 研究并提出了一种基于 “ $3 \mathrm{~S}$ ” 架构的自动光网络 (autonomous optical networks, AON), 实现网络状态 的自感知 (self-aware)、网络控制的自适应 (self-adaptive) 和网络运行的自管理 (self-managed), 将 AI 技术引入自动光网络中, 在器件、链路和组网等多个层面有效提升 $\mathrm{AON}$ 性能, 实现光网络的自治.

“An overview of ML-based applications for next generation optical networks" 回顾了可用于光网络 的机器学习 (machine learning, ML) 技术. 聚焦网络设计、功率优化、路由和波长分配以及错误管理 等关键挑战, 并讨论了技术演进的方向.

"AI based on frequency slicing deep neural network for underwater visible light communication" 研 究并提出了一种低复杂度频率切片深度学习网络 (frequency slicing deep neural network, FSDNN), 解 决水下可见光通信系统中的宽带信号后置补偿问题. 
"Intent defined optical network with artificial intelligence-based automated operation and maintenance" 研究并提出了意图定义光网络 (intent defined optical network, IDON) 中基于 AI 的自动运行 和维护技术, 利用自适应生成和优化策略 (self-adopted generation and optimization, SAGO) 完成服务 目标.

"Overfitting effect of artificial neural network based nonlinear equalizer: from mathematical origin to transmission evolution” 研究了光纤通信系统中基于 AI 技术的非线性均衡器面临的过拟合问题, 并 讨论了相关的技术途径和解决方案.

$\mathrm{AI}$ 驱动的光通信专题主要面向光通信、人工智能及相关领域的研究人员, 反映了该技术领域的一 些前沿方向与研究进展, 希望能够对 AI 驱动的光通信领域的研究工作有所促进. 